\title{
Interfacial Dissipative Phenomena in Tribomechanical Systems
}

\author{
Antonio Papangelo ${ }^{1,2}$ (D)
}

1 Department of Mechanics Mathematics and Management, Politecnico di Bari, Via Orabona 4, 70125 Bari, Italy; antonio.papangelo@poliba.it

2 Institute of Structural Dynamics, Am Schwarzenberg-Campus 1, Hamburg University of Technology, 21073 Hamburg, Germany

Keywords: friction; adhesion; dissipation; nonlinear dynamics; contact nonlinearities

Citation: Papangelo, A. Interfacial Dissipative Phenomena in

Tribomechanical Systems. Lubricants 2021, 9, 104. https://doi.org/ 10.3390/lubricants9100104

Received: 8 October 2021

Accepted: 11 October 2021

Published: 15 October 2021

Publisher's Note: MDPI stays neutral with regard to jurisdictional claims in published maps and institutional affiliations.
The last decade has experienced a tremendous development of several technologies that are likely to shape our future. These comprise soft robots, humanoids, autonomous driving, aeronautic, and space technologies. To reduce the development costs, the dynamical behaviour of machines, usually consisting of several jointed components, is often simulated numerically. To ensure a good matching between numerical and experimental results it is of utmost importance to implement reliable and resilient models, which accurately describe the interactions among the different parts of the machine. Indeed, dynamicists are aware that the most difficult part of simulating structure dynamical behaviour is a good understanding of its damping properties. Ensuring a certain service life often coincides with taking care that the vibration amplitude remains below a certain admissible threshold so that the component can confidently fulfil its task along with the machine lifespan. To increase the machine damping properties, dampers are accurately positioned in the structure so that they dissipate as much energy as possible, particularly when the machine is excited close to one of its natural frequencies. Nevertheless, mechanical structures are almost always constituted by several jointed components, hence, a significant share of the energy is dissipated at the contact interfaces. Riveted and bolted joints, seals and bearings all significantly contribute to the damping properties of the assembly and to its dynamical behaviour due to the introduction of nonlinear interaction forces. Nevertheless, after scrutiny of the relevant engineering literature, one finds out that scientific contributions from tribologists rarely take into account the effect of structure dynamic excitation and, on the other hand, dynamicists very often include interfacial characteristics through very simplified empirical models such as the Coulomb friction coefficient and linear contact stiffness.

This Special Issue (SI) was conceived with the intent of encouraging researchers to submit scientific contributions where the interplay between dynamics and tribology is evident. I am pleased to see that this Special Issue has collected seven scientific articles and one review article, all pertaining to the themes of tribology and dynamics.

In Ref. [1] Bonari and Paggi have developed a novel interface finite element procedure to deal with the normal and tangential contact problem of a rigid indenter with an arbitrary profile and a viscoelastic substrate. Through numerical simulations, they have shown that the effect of Coulomb friction and viscoelastic dissipation can be simultaneously investigated, leading to an accurate estimate of the surface tractions, hence, of the energy that is dissipated through the contact interface.

Ref. [2] and [3] have focused on a different source of dissipation, namely that which occurs due to interfacial adhesion. It is well-known from physics that contacting bodies interact through short-range repulsive and long-range adhesive interactions (van der Waals forces). Commonly, adhesion at macroscopic scales is not observed as interfaces are generally randomly rough. By exploiting a multi-asperity representation of a self-affine randomly rough interface with Gaussian distribution of heights, Violano and Afferrante [2] 
have studied the loading and unloading behaviour of a rough interface, particularly focusing on how the pull-off detachment force and the energy dissipation depend on the surface characteristics. Remarkably, they have shown that dissipation in a loading cycle is proportional to the real contact area, which is in agreement with other analytical [4] and experimental [5] results. Furthermore, they found hysteretic dissipation and pull-off force are much more scattered for surfaces with high fractal dimension. Although roughness, and in particular its height root mean square, is detrimental to macroscopic adhesion, there exist special topographies [6-8] that can be exploited to enhance macroscopic adhesion. Papangelo and Ciavarella in Ref. [3] have used the Boundary Element Method to study the adhesive behaviour of a sphere with superposed an axisymmetric single-wavelength roughness. This geometry has been studied by Guduru and collaborators both analytically [6] and experimentally [7], showing that the pull-off force increases by a factor of about 20 with respect to the smooth sphere. For the Guduru theory to be valid, the contact patch should be simply connected, without inner cracks. In Ref. [3] the contact problem has been solved numerically, also investigating the regions where the theory's hypotheses were not valid. It was shown that the highest pull-off force is reached for a ratio $\mathrm{A} / \lambda \approx 10^{-1}$ (A and $\lambda$ being, respectively, the waviness amplitude and wavelength) as lower values tend to the smooth sphere behaviour with small adhesion enhancement and larger values tend to the appearance of external cracks.

Reference [9] by Zeng and Qi is a good example of how tribological problems are indeed multiphysics, involving several lengths and time scales. Zeng and Qi [9] have studied the process of erosion and corrosion in pipes that transport two-phase flow (gassolid) encountering a pipe elbow. They have used a multiphysics finite element simulation software to couple turbulent fluid dynamics for the flow, chemical reactions for corrosion and particle dynamics for erosion. Their results show that marked erosion mechanisms are at play on the elbow extrados surface at $40-50^{\circ}$. Furthermore, turbulence influences the concentration of substances, which plays a role in the rate chemical reactions take place (corrosion).

Reference [10] by Genovese et al. is a review on friction and wear test rigs. It is a comprehensive collection of working principles and specifics that are in use to objectively measure friction and wear data. Starting from the first concepts of Leonardo da Vinci, dating back to the 15th century, nowadays different solutions exist, which allow imposing a certain normal load and a relative velocity to two mating interfaces. As noted, only few friction testers allow for temperature control, lubricated or dry contact conditions and permit outdoor measurements to be conducted in real scenarios.

In Ref. [11] Nouira et al. have numerically and experimentally studied the propagation of elastic waves in a mechanical assembly that presents contact interfaces. Indeed, due to the interfacial roughness, the contact stiffness is nonlinear, often represented with a power-law model, which introduces higher-order harmonics into the system dynamical response. The authors have considered the case of two contacting bodies excited by an impulsive force, which was modelled numerically and compared with experimental measurements. Using different contact laws, the authors have shown that accurately modelling the interfacial contact stiffness is crucial to correctly predict the system nonlinear oscillations, proving once more the tight link between tribology and dynamics.

References [12] and [13] dealt with the problem of friction-induced vibrations, which may trigger tedious noise in the proximity of the sliding interface. There are several mechanisms that may trigger friction-induced vibrations; among others, a decaying characteristic of the friction law with sliding velocity. In Ref. [12] Hu and Habib have considered the use of a dynamic vibration absorber (DVA) to suppress undesired vibrations. They have shown that the character of the bifurcation (sub- or supercritical) can be controlled by a proper design of the nonlinear restoring force of the DVA, although the best performance, in terms of reducing the velocity range where stick-slip vibrations occur, is obtained by using a DVA with linear stiffness. Reference [14], by Stender et al., focused on the concept of basin stability in nonlinear systems. Indeed, the classical concept of linear stability is of 
little help in nonlinear systems where several solutions may coexist in the same parametric region. Linear stability is, in fact, limited to small perturbations around the equilibrium position. On the contrary, given a finite region in the state space where initial conditions are expected to lie, basin stability gives the likelihood for a dynamical system to converge to a certain equilibrium state (static or dynamic). This concept was successfully applied to frictional oscillators that present multiple co-existing stable solutions and proved to be a valuable tool for the analysis of multi-stable systems, particularly when operating conditions are well known.

Finally, the Guest Editor would like to express his sincere gratitude to all authors and reviewers who contributed to this Special Issue and to the editorial staff of Lubricants for their valuable support.

Funding: This research received no external funding.

Institutional Review Board Statement: Not applicable.

Informed Consent Statement: Not applicable.

Conflicts of Interest: The author declares no conflict of interest.

\section{References}

1. Bonari, J.; Paggi, M. Viscoelastic Effects during Tangential Contact Analyzed by a Novel Finite Element Approach with Embedded Interface Profiles. Lubricants 2020, 8, 107. [CrossRef]

2. Violano, G.; Afferrante, L. Roughness-Induced Adhesive Hysteresis in Self-Affine Fractal Surfaces. Lubricants 2021, 9, 7. [CrossRef]

3. Papangelo, A.; Ciavarella, M. A numerical study on roughness-induced adhesion enhancement in a sphere with an axisymmetric sinusoidal waviness using Lennard-Jones interaction law. Lubricants 2020, 8, 90. [CrossRef]

4. Deng, W.; Kesari, H. Depth-dependent hysteresis in adhesive elastic contacts at large surface roughness. Sci. Rep. 2019, 9, 1-12.

5. Dalvi, S.; Gujrati, A.; Khanal, S.R.; Pastewka, L.; Dhinojwala, A.; Jacobs, T.D. Linking energy loss in soft adhesion to surface roughness. Proc. Natl. Acad. Sci. USA 2019, 116, 25484-25490. [CrossRef] [PubMed]

6. Guduru, P.R. Detachment of a rigid solid from an elastic wavy surface: Theory. J. Mech. Phys. Solids 2007, 55, 445-472. [CrossRef]

7. Guduru, P.R.; Bull, C. Detachment of a rigid solid from an elastic wavy surface: Experiments. J. Mech. Phys. Solids 2007, 55, 473-488. [CrossRef]

8. Papangelo, A.; Ciavarella, M. A Maugis-Dugdale cohesive solution for adhesion of a surface with a dimple. J. R. Soc. Interface 2017, 14, 20160996. [CrossRef] [PubMed]

9. Zeng, Q.; Qi, W. Simulation Analysis of Erosion-Corrosion Behaviors of Elbow under Gas-Solid Two-Phase Flow Conditions. Lubricants 2020, 8, 92. [CrossRef]

10. Genovese, A.; D'Angelo, G.A.; Sakhnevych, A.; Farroni, F. Review on friction and wear test rigs: An overview on the state of the art in tyre tread friction evaluation. Lubricants 2020, 8, 91. [CrossRef]

11. Nouira, D.; Tonazzi, D.; Meziane, A.; Baillet, L.; Massi, F. Numerical and experimental analysis of nonlinear vibrational response due to pressure-dependent interface stiffness. Lubricants 2020, 8, 73. [CrossRef]

12. Hu, J.L.; Habib, G. Friction-Induced Vibration Suppression via the Tuned Mass Damper: Optimal Tuning Strategy. Lubricants 2020, 8, 100. [CrossRef]

13. Stender, M.; Hoffmann, N.; Papangelo, A. The basin stability of bi-stable friction-excited oscillators. Lubricants 2020, 8, 105. [CrossRef]

14. Stender, M.; Hoffmann, N. bSTAB: An open-source software for computing the basin stability of multi-stable dynamical systems. Nonlinear Dyn. 2021, 1-18. [CrossRef] 